marked impairment of hearing, the ear trouble may not be suspected, and it is only after inflation that the child feels that its ears are now " more natural" than they were before. Such slight " Eustachian obstruction, and the headaches dependent thereon, can be readily cured in children by a few inflations, either by Pollitzer's method or, still better, by means of the Eustachian catheter. The naso-pharynx should, of course, receive due attention, in order to prevent a return of the Eustachian obstruction.

Centra1 Music Hall, Chicago.

\section{A SYSTEM OF FREE NURSING AS OR- GANIZED IN PHILADELPHIA.}

Read in the Section on Obstetrics and Gynecology at the Thirty-ninth Annual Meeting of the American Medical Association, Cincinnati, May, 1888 .

BY JOSEPH PRICE, M.D., OF PHILADELPHA.

I desire to call the attention of this Association to this system of free nursing because I regard it as a most valuable factor in the proper treatment of the sick poor; as a necessary lesson to the lower classes in cleanliness and the care of the sick; as the most efficient aid to the surgeon in non-paying practice, and because $I$ hope that the idea suggested may prove to be of practical benefit to the profession in cities and towns not blessed with suitable institutions for the proper care of the sick poor.

The Visiting Nurse Society, of Philadelphia, was organized a little over two years ago by a few charitable ladies of this city for the purpose, as their charter reads, "of furnishing visiting nurses to those unable to secure skilled attendance in time of illness, to teach cleanliness and the proper care of the sick." It is supported by voluntary contributions and such small amounts as the patients may be able to pay, and its last report shows a remarkable amount of practical benevolence secured by the outlay of a very small sum of money. The Society in the beginning employed a trained nurse with one or two assistants, who were also pupils. Additional nurses were employed as necessity arose until the staff now consists of seven nurses and assistants and one maternity nurse whose work is confined to that specialty. Special nurses are provided for contagious diseases as they may be required, the regular staff not coming in contact with such cases. The Society was very fortunate in the selection of their head-nurse, who is a trained nurse of more than ordinary ability, both in practicing and teaching her profession.

The assistants are all young, healthy women, selected for their peculiar qualifications and carefully trained under the supervision of the head nurse. In cases demanding immediate operation these nurses make all preparations at the shortest possible notice, viz. : thorough cleansing of the room and person of the patient, often supplying fresh body and bed linen, one or more nurses to assist in the operation, and one to care for the patient during the subsequent treatment. I have known these nurses to go in an attic or cellar in the heart of the slums of the city, the rooms reeking with filth and overrun with vermin. The patients, fit inhabitants of their homes, destitute of the bare necessities of life, not having even a receptacle in which to boil water, and often dependent on their neighbors for food and fuel. In a few hours the nurses have cleaned the room, supplied the necessary funiture and utensils and prepared the patient for an abdominal section. With such an organization at his command the physician or surgeon has no reason to fear undertaking any case, surgical or medical, at the homes of even the poorest of patients. In the clinic of the female department of the Philadelphia Dispensary, for years I did not attempt to do any of the abdominal work that constantly presented itself because of the lack of such a free system of trained nurses.

Since the organization of this Society we have done over ninety abdominal sections in the alleys and courts of this city, with only one death. And these were not selected cases, but were done because they were imperative. In addition, many general operations of more or less severity, but requiring skilled nursing, have been performed, and in every case the nurses of this Society have proved themselves equal to the occasion. During the past year the Society has cared for 90 surgical cases, out of a general list of 369 , necessitating nearly 6000 visits. So far during this year they have attended nearly 200 cases, of which 50 per cent. were surgical.

The medical profession of Philadelphia has reason to congratulate itself in possessing such an efficient corps of assistants, and to wish for the extension of the benevolence to localities less favored.

\section{CALCIFICATION OF THE CARDIAC VALVES. DEATH FROM CERE- BRAL EMBOLUS.}

Real before the Medical Society of the Distria of Columbia, Aprit IT, 1888 .

BY FREDERICK SOHON, M.D., OF WASHINGTON, D. C.

The calcification of the cardiac valves, shown in the specimen presented to-night at the request of several members who have already examined it, derives its interest not from the simple fact that it shows a diseased organ, but from the circumstances attending the patient's life and the manner of his death.

His family and friends give the same account 